\title{
Local treatment of liver and lung metastases from colorectal cancer: a multicenter Tunisian study
}

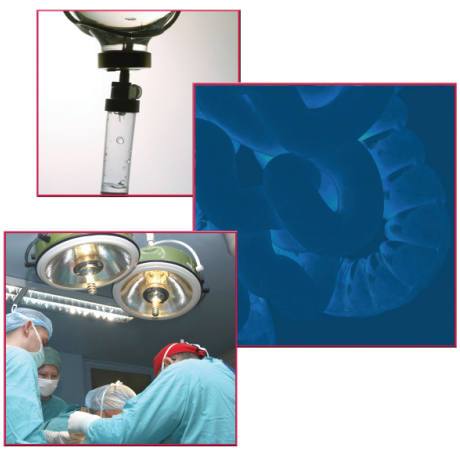

Haifa Rachdi*, ${ }^{1}$, Soumaya Labidi ${ }^{1}$, Nesrine Mejri ${ }^{1}$, Houda El Benna ${ }^{1}$, Nouha Daoud ${ }^{1}$, Rached Bayar ${ }^{2}$, Adel Marghli ${ }^{3}$, MedTahar Khalfallah² \& Hamouda Boussen ${ }^{1}$

${ }^{1}$ Medical Oncology Department (SOMA), Abderrahmen Mami Hospital, Ariana, Tunisia

${ }^{2}$ Hepatobiliary Surgical Department, Mongi Slim Hospital, La Marsa, Tunisia

${ }^{3}$ Thoracic Surgery Department, University Hospital Abderrahmen Mami Hospital, Ariana, Tunisia

*Author for correspondence: Tel.: +216 5886 2984; haifa.rachdi@yahoo.fr

\begin{abstract}
Aim: Surgical treatment of hepatic or pulmonary metastases is the optimal therapeutic goal in metastatic colorectal cancer (CRC). Methods: Our retrospective study concerned 70 patients treated for CRC, collected from 2003 to 2015, presenting liver metastases (LM) in 61 cases and pulmonary metastases (PM) in nine cases, treated by surgery for their distant disease. We collected and compared their epidemiologic, anatomoclinical parameters and analyzed several prognostic factors. Results: Metastases were multiple $(\geq 4)$ in 9/61 LM and in 5/9 PM. Patients had synchronous metastases in 32 cases (30 LM/2 PM) and metachronous metastases in 33 cases (32 LM and 11 PM). Surgery for LM consisted of metastasectomy (49/61), segmentectomy $(5 / 61)$ and hepatectomy for the remaining seven patients; ten patients had also subsequent liver radiofrequency. LM were treated by wedge resection in $6 / 9$ and lobectomy in two cases, radiofrequency was performed in five cases. 56/61 (80\%) patients received chemotherapy, mostly FOLFOX protocol as the first-line treatment and targeted therapy in $55 \%$ of cases. For the overall population, median overall survival (OS) and progression-free survival (PFS) were, respectively, 44 and 32 months. We did not observe any significant difference in terms of OS $(p=0.659)$ and PFS $(p=0.318)$ between resected LM or/and PM. A better survival was found when there was disease-free interval between the occurrence of the primary and the metastases exceeded 18 months and in patients with less than four metastases. Conclusion: Resection of metastatic disease mostly in liver and lungs improves survival of patients with CRC. The patients with longer disease-free interval and less than four metastases had the best outcomes.
\end{abstract}

First draft submitted: 15 February 2018; Accepted for publication: 20 December 2018; Published online: 2 April 2019

Keywords: cancer $\bullet$ colorectal $\bullet$ hepatic $\bullet$ metastases $\bullet$ pulmonary $\bullet$ surgery $\bullet$ suvival

Colorectal carcinoma (CRC) has increased in incidence over the past 20 years worldwide and in Tunisia [1,2]. A recent study concerning the center of Tunisia, evaluated the incidence of CRC during the period, 1993-2007, and showed that it increased by $+2.6 \%$ and $+5.3 \%$ for females and males [1]. After curative surgery, CRC remains a disease with a high relapse risk; $50 \%$ of patients would develop metastases, mostly in the liver (33\%) and lungs in $22 \%[3]$. Without treatment, metastases impairs prognosis, decreasing the chances of 5 -year survival to less than $5 \%[5,6]$.

During the last 10 years, a multidisciplinary team (medical oncologists, surgeons, radiolgists and histopathologists) for the management of CRC has become mandatory [7]. The combination of systemic chemotherapy and targeted therapy can increase objective response rate in metastatic sites, like the liver and lungs and allows more possibilities of local treatment by surgery and/or interventional radiology [8]. Nowadays, surgical resection and/or interventional radiology for hepatic metastases, improves the chances of 5-year survival to 39\% [9]. Likewise, complete resection of lung metastases also improved the 5-year survival from 21 to $43 \%$ [10]. On the other hand, a one-time surgery for both LM and PM may have a different impact on survival versus surgery for only one anatomic site [11].

Future : Medicine 
The aim of our study was to report epidemiologic and clinical features of patients operated on for LM and PM from CRC and to analyze outcome and prognostic factors.

\section{Methods}

We retrospectively analyzed patients who underwent radical (R0/R1) resection of LM or PM, from 2003 to 2015. Metastases were either synchronous (within 3 months after primary tumor resection) or metachronous, after a disease-free interval of more than 3 months. Initial work-up included: anamnesis, clinical exam, biology (tumor markers: CEA), RAS oncogene status (mutated or wild), colonoscopy, thoraco-abdomino-pelvic computerized tomography scan, pelvic magnetic resonance imaging (rectal cancer). No patient underwent a positron emission tomography scan because of its unavailability in our country. All patients were treated according to the decisions of a multidisciplinary tumor board, involving medical oncologists, surgeons, gastroenterologists, radiologists and histopathologists. Performed liver resections were nonanatomical (solitary or multiple wedge resection), or anatomical (lobectomy, segmentectomy or hemi-hepatectomy). The approach of resection was either laparotomic or laparoscopic. Additional radiofrequency ablation was also discussed in combination with liver or pulmonary resection if surgery was not possible or for small lesions. Performed pulmonary resections were wedge resection, segmentectomy and bilateral resections of PM. Recurrent lesions after previous surgery for metastases were not a contraindication for further resection. The clinical approach becomes progressively more radical over the time. Chemotherapy associated or not to targeted therapies are used before and after surgery according to decisions of multidisciplinary tumor board. Patients were evaluated clinically, with tumor markers and according to radiologic The Response Evaluation Criteria in Solid Tumors (RECIST) criteria, and underwent follow-up every 3 months with repeated scans and monitoring tumor markers. We also assessed overall and disease-free survival. The start date of survival was the date of diagnosis. We evaluated the prognostic value of several parameters: age, sex, number of metastases, disease-free interval (DFI), perioperative chemotherapy and targeted therapy.

\section{Statistics}

Statistical analysis was performed using SPSS 17.0 program. Comparison between patient subgroups was performed using Pearson's chi-square test. Actuarial survival analysis was calculated by Kaplan-Meier method, comparisons with log-rank test. Cox regression model was used for the multivariable model; the date point for DFI was the date of primary tumor resection.

\section{Results}

Out of 350 patients with metastatic colon cancer (liver and or lung), 70, presenting LM (61) and PM (9) locally treated were identified during the study period. Patients who underwent resection in other centers and those who were lost to follow-up were excluded.

Metastases were multiple ( $\geq 4)$ in 9/61 LM and in 5/9 PM. Patients had synchronous lesions in 32 cases (30 $\mathrm{LM} / 2 \mathrm{PM}$ ) and metachronous in 38 cases (32 LM/7 PM). Resection of primary tumor was done in 30 cases. The sex ratio was 3.11, median age at diagnosis was 55 years (32-78 years), 9/70 were aged under 40 years. Primary tumor was located in the right colon in 39\%, in left colon in $16 \%$ and in the rectum in $17 \%$ of cases. Tumor marker (CEA and/or CA 19-9) levels were initially high in 61\% of cases. Primary CRC was classified as T2 (15.7\%), T3 (50\%) and T4 (34.3\%) and satellite lymph nodes were histologically invaded in $82 \%$ of cases (Table 1). RAS oncogene was wild in $57 \%$ and mutated in $10 \%$ of cases and not evaluated in the remaining $33 \%$ of cases. 56/70 (80\%) patients received perioperative chemotherapy, mostly with FOLFOX regimen in $86 \%$ of cases. Postoperative chemotherapy was administered in $48 \%$ of patients. Half of the patients received targeted therapy; bevacizumab in $45 \%$ and cetuximab in $5 \%$ of cases. Response rates were: $81 \%$ of objective responses, $4 \%$ of stable disease and $15 \%$ disease progression. Liver surgery for metastases concerned 61 patients, lung surgery for nine patients and both in five patients. Margins of resection were R0 in 57 of cases and R1 in 13 of cases. Surgery for LM consisted of metastasectomy (49/61), segmentectomy (5/61) and hepatectomy $(7 / 61)$, and 11 patients also had subsequent liver radiofrequency. PM were treated by wedge resection in 6/9 cases and lobectomy in three cases, additional radiofrequency was performed in five cases (Table 2).

Median PFS and OS were 32.4 and 44 months, respectively. There was no significant difference found in terms of survival between patients with LM alone, PM alone or both sites ( $p=0.659$ for OS and $p=0.318$ for PFS; Figures $1 \& 2$ ). For resected LM, an overall survival rate at 1,2 and 3 years was estimated at 80,60 and $53 \%$ for resected LM, respectively, while it was $80 \%$ at 1 year, $60 \%$ at 2 years and $51 \%$ at 3 years for resected PM. 
Table 1. Patient characteristics.

Data

$n(\%)$

Age

$\leq 40$ years

$\begin{array}{ll}\text { Sex-ratio } & 3.11\end{array}$

Primary site

Right colon 27 (39)

Left colon 11 (16)

Rectum 17 (24)

Synchronous metastases

$\begin{array}{ll}\text { Hepatic } & 30(48)\end{array}$

Lung 2 (15)

\section{Metachronous metastases}

Hepatic $32(52)$

Lung 11 (84)

Hepatic metastases $\quad 62(89)$

$124(38)$

$2-3 \quad 29(48)$

$\geq 4 \quad 9$ (15)

$\begin{array}{ll}\text { Lung metastases } & 10(7)\end{array}$

$<3 \quad 6(60)$

$\geq 4 \quad 4(40)$

Lung + hepatic metastases $\quad 5(7)$

T stage

T1 0

T2 11 (15.7)

T3 $35(50)$

T4 24 (34.3)

KRAS-mutation status

\begin{tabular}{|ll|}
\hline Mutated & $40(57)$ \\
\hline Wild & $7(10)$ \\
\hline Not done & $23(33)$ \\
\hline
\end{tabular}

PFS at 1, 2 and 3 years, was 58, 48 and 30\% for LM, respectively. It was $70 \%$ at 1 year, $40 \%$ at 2 years and $34 \%$ at 3 years for resected PM. For patients who had both LM and PM resection, OS was $82 \%$ at 1 year and $60 \%$ at 2 years and $53 \%$ at 3 years. PFS was estimated at $72 \%$ at 1 year, $50 \%$ at 2 years and $37 \%$ at 3 years.

Univariate analysis showed nonsignificant trend for improved survival according to gender $(p=0.09)$. The number of liver metastases $(<4)$ and a DFI > 18 months had significant impact on OS and PFS (Table 3).

\section{Discussion}

We report a group of Tunisian series of patients with LM (61/70) and PM (9/70), treated by surgical resection, indicated by a multidisciplinary team for their metastatic disease. Treatment strategy: medical regimens and timing of surgery (initial or delayed after chemo and/or targeted therapy) were always discussed within a multidisciplinary approach. Surgery was mostly indicated in patients presenting initially with resectable lesions or with a good response after initial medical therapy. It is clearly proved that initial or delayed complete resection of LM and PM from CRC, combined with medical therapies (chemotherapy and targeted therapies), improved the 5-year survival with rates reaching 30-39\% in selected patients with LM and also 30-60\% in case of PM [4,6,8-10,14-16,19] We observed in our population a 5 -year survival of $32 \%$ after liver metastasectomy and $42 \%$ after pulmonary metastasectomy, without significant difference $(\mathrm{p}=0.659)$. Two small series of young patients treated with an aggressive liver and lung surgery reported a 5-year survival of 68.6 and 77\% [19,20]. Elias et al. [20] suggest that a prolonged survival can be achieved after concomitant or in two-times surgeries for their metastases. Conversely, Gough et al. reported that OS after resection of both LM and PM was worse than after resection for PM only (11 vs 27\% OS at 5 years) [11]. 
Table 2. Medical protocols, response and surgical treatment.

\begin{tabular}{|c|c|}
\hline Treatment & $n(\%)$ \\
\hline Perioperative chemotherapy & $56(82)$ \\
\hline FOLFOX & $51(86)$ \\
\hline LV5FU2 & 1 (2) \\
\hline FOLFIRI & $7(12)$ \\
\hline \multicolumn{2}{|l|}{ Targeted therapy } \\
\hline Avastin & $33(50)$ \\
\hline No & $30(45)$ \\
\hline \multicolumn{2}{|l|}{ Response } \\
\hline SD & $2(4)$ \\
\hline PR & $44(81)$ \\
\hline PD & $8(15)$ \\
\hline Lobectomy & $0(0)$ \\
\hline Right hepatectomy & $2(3)$ \\
\hline Left hepatectomy & $5(8)$ \\
\hline Radiofrequency for liver metastases & $11(17)$ \\
\hline \multicolumn{2}{|l|}{ Surgery for lung metastases } \\
\hline Lobectomy & $2(20)$ \\
\hline Segmentectomy & $0(0)$ \\
\hline Wedge & $6(60)$ \\
\hline Radiofrequency for lung metastases & $5(50)$ \\
\hline \multicolumn{2}{|c|}{ PR: Partial response; PD: Progressive disease; SD: Stable disease. } \\
\hline
\end{tabular}

In our study, both hepatic and pulmonary resections were performed in only 5/70 patients with an OS of $72 \%$ at 1 year and $37 \%$ at 3 years. Surgery of metastatic disease is difficult and unfeasible in a high number of patients, due to patient's condition-related contraindications and a lack of multidisciplinary boards and teams with experienced and subspecialized surgeons and also lack of interventional radiologists. The use of stringent selection criteria for surgical approach will reasonably increase the resection rate and chance of curing LM and PM from CRC [20-23]. Metastasectomy has to be as complete as possible with an objective of R0 resection [24]. Work-up has to eliminate the presence of extra liver and/or lung metastatic sites, such as peritoneal carcinosis, hepatic or celiac metastatic lymph nodes, a common contraindication of surgery. However multifocal LM and/or PM requiring multiple surgeries, should not be a contradiction to surgical treatment. Vogt et al. [25]. reported no significant difference of survival in patients with a unique versus multiple PM, whereas the number of resected LM did not influence the outcome. We performed in our series the resection of multiple metastases $(\geq 4)$ in nine patients with LM and in four patients with PM. In our study, the number of metastases is considered as a significant prognostic factor for OS ( $\mathrm{p}=0.027)$ and PFS ( $p=0.042)$. Furthermore, a DFI $>18$ months was found in the series of Limmer et al. [12]. to be a significant prognostic factor of OS. A prolonged DFI was one of the most important factors for OS. Furthermore, in the series of Regnard et al. [24] and concerning pulmonary metastases, DFI was a significant prognostic factor with a better survival for patients with metachronous pulmonary metastases; this result was in accordance with the series of Van Halteren and coworkers [26]. In our study, patients with a DFI of more than 18 months had a significant impact on OS and PFS versus patients with a DFI $<18$ months with significant $p$-value $(\mathrm{p}=0.31$ for OS and $\mathrm{p}=0.045$ for PFS).

Other prognostic variables such as CEA level or number of lesions are also known to affect survival, but none of them are generally accepted to be an absolute contraindication for surgical resection. Headrick et al. [27] reported the presence of lymph node metastases within the chest as one possible contraindication to surgery. They identified four patients in their series with positive thoracic lymph nodes who demonstrated a short DFI and a limited 


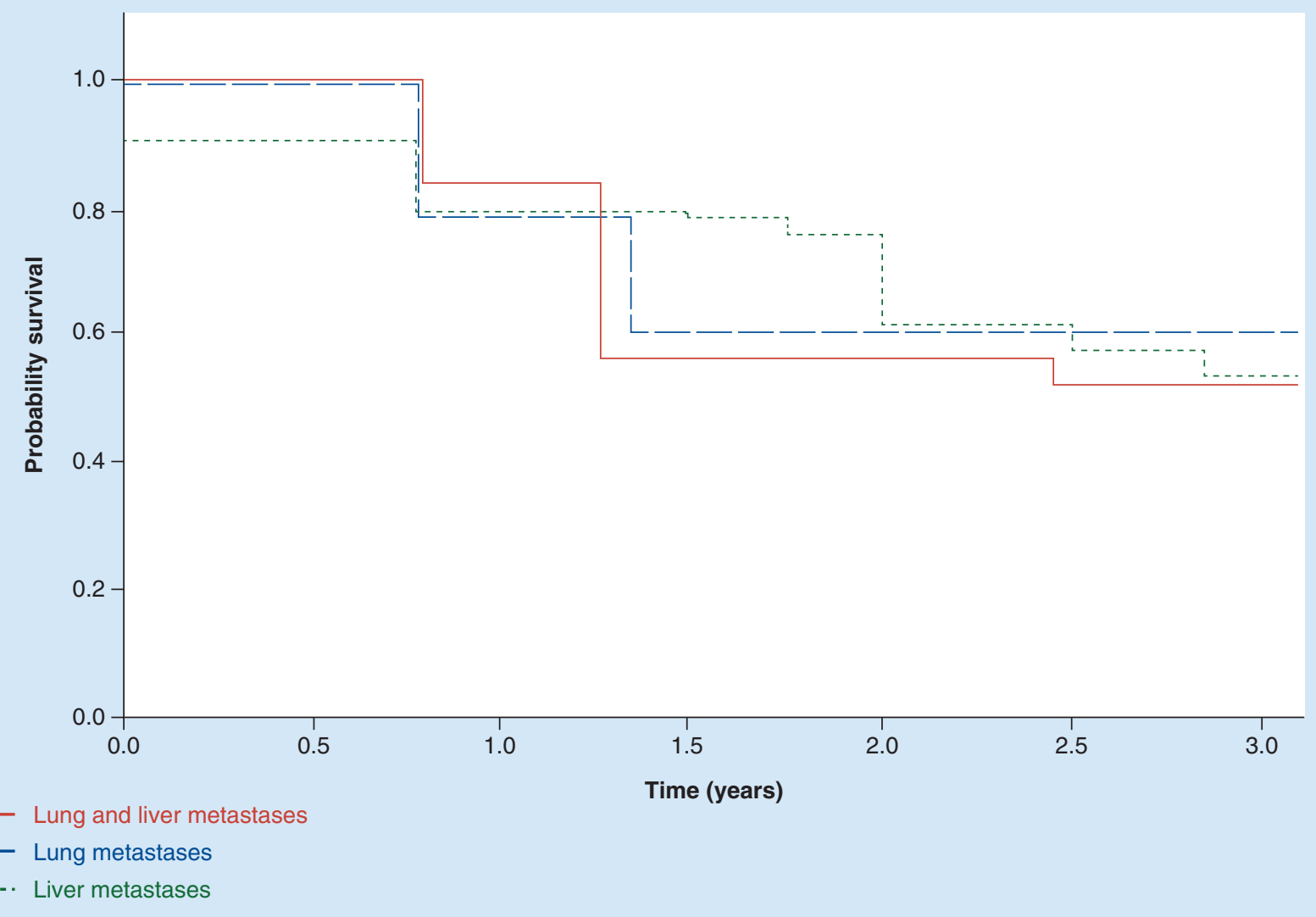

Figure 1. Overall survival.

Logrank test $p$-value $=0.659$.

survival. These results were trend-setting but not statistically significant. Macherey et al. have described in their study that number, size and distribution of PM have limited prognostic value, compared with the high CEA level before surgery and presence of intrathoracic lymph node metastases [10].

Lung and/or liver resection, combined with chemo and/or targeted therapy is nowadays still the only curative treatment for selected patients with metastatic CRC $[7,12,17,18]$. New surgical techniques, increased the pool of resectable patients with colorectal LM or PM, for example, for patients with LM, a two-stage hepatectomyassociated liver partition and portal vein ligation for staged hepatectomy and ultrasound-guided enhanced one-stage hepatectomy [13,14].

Sequential resection of LM and PM should be carried out whenever possible. In the study of Adam et al. [28], sequential resection of LM and PM was performed in 150 patients with metastatic CRC. The median number of liver and lung metastases resected was 3 and 1, respectively. They found that a high level of CEA before lung surgery $>100 \mathrm{ng} / \mathrm{ml}(\mathrm{p}=0.014)$ and an interval between liver and lung resection of $<24$ months $(\mathrm{p}=0.024)$ were independent poor prognostic factors. Surgery may improve the prognosis of some patients with unresectable metastases, who responded to medical treatment, with the same prognosis of those with initially resected disease, stressing on the importance of local treatment [29,30]. In contrast, combined resection + radiofrequency ablation (RFA) did not offer a therapeutic benefit for high-risk patients with two or more risk factors [31]. These findings may serve as a basis for improved selection of patients with borderline resectable or unresectable lesions being considered for resection plus ablation.

From a surgical point of view, every attempt must be made to increase the availability of any liver or lung resection or both in order to improve survival rates of patients with MCRC $[30,31]$.

Based actually to the ESMO recommendation of 2016 [32], surgical and oncological criteria should be considered to discuss hepatic or pulmonary metastases in CRC: 


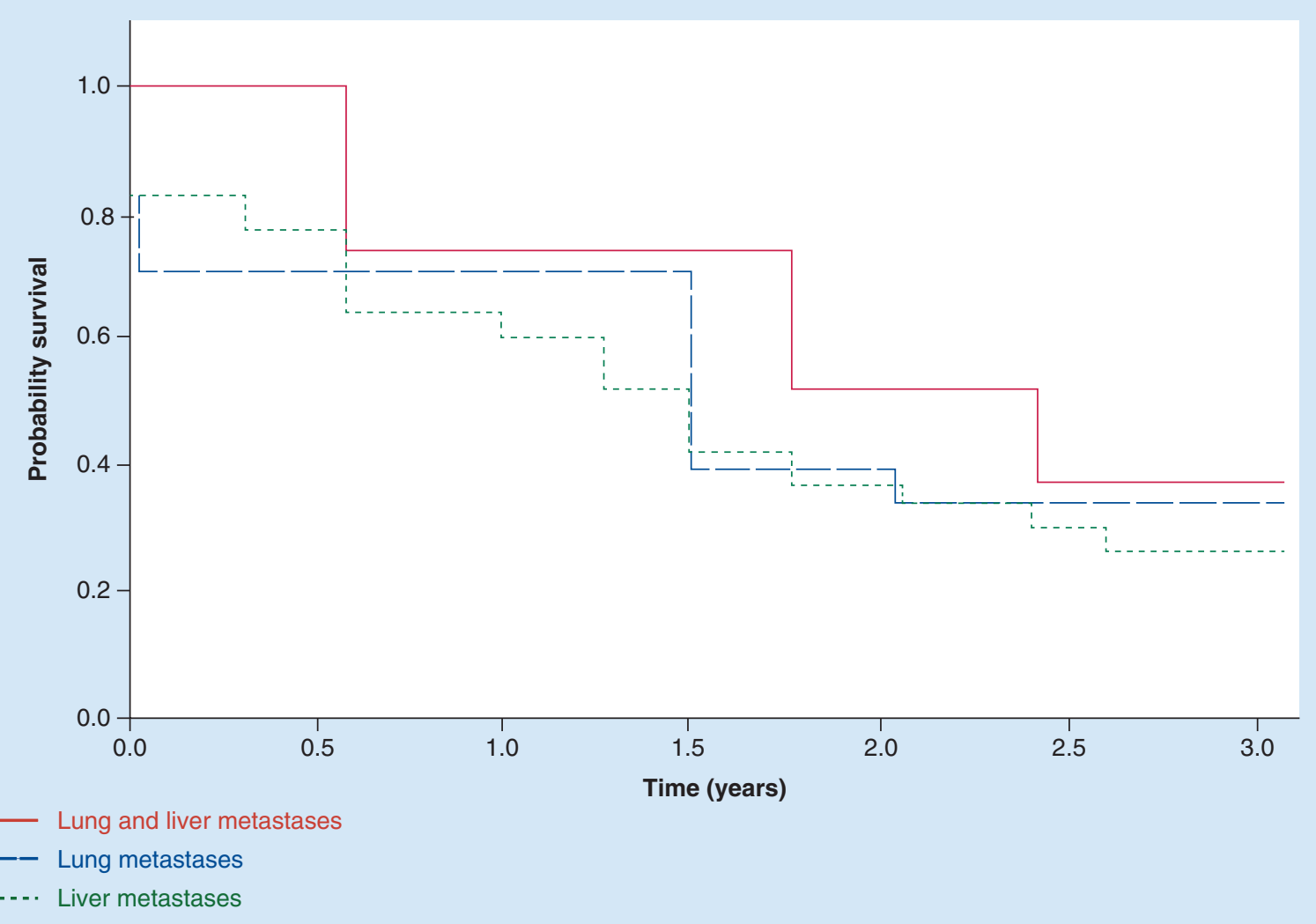

Figure 2. Progression-free survival. Logrank test $p$-value $=0.318$.

- Patients with excellent oncological criteria and easy technical criteria immediately have conducted to hepatic or pulmonary resection. Adjuvant chemotherapy with folfox regimen can be discussed.

- Patients with good oncological criteria and easy technical criteria should be treated with perioperative chemotherapy.

\section{Conclusion}

Resection of metastatic disease identified in the liver and lungs improves survival of patients with a CRC. Patients with longer disease-free interval and less than four metastases have the best outcomes.

\section{Executive summary}

- Colorectal carcinoma has increased in incidence over the past 20 years worldwide and in Tunisia.

- Surgical treatment of hepatic or pulmonary metastases is the optimal therapeutic goal in metastatic colorectal cancer (CRC).

- Our study aims to report and compare epidemiologic, anatomo-clinical and prognostic factors in patients treated by surgery for their liver and lung metastases.

- It is a retrospective study that concerned 70 patients treated for CRC, collected from 2003 to 2015, presenting liver metastases in 61 cases and pulmonary metastases in nine cases, treated by surgery for their distant disease.

- Resection of metastatic disease mostly in liver and lungs improves survival of patients with CRC. Patients with longer disease-free interval and less than four metastases had the best outcomes. 
Table 3. Potential prognostic factors tested by univariate analysis.

\begin{tabular}{|c|c|c|c|c|c|c|}
\hline \multirow{2}{*}{ Characteristics } & \multicolumn{3}{|c|}{ PFS } & \multicolumn{3}{|c|}{ os } \\
\hline & HR & $95 \% \mathrm{Cl}$ & p-value & HR & $95 \% \mathrm{Cl}$ & p-value \\
\hline Gender & & $0.86 ; 1.74$ & 0.265 & & $0.93 ; 2.45$ & 0.097 \\
\hline Female & 1 & & & 1 & & \\
\hline Male & 1.22 & & & 1.51 & & \\
\hline Age & & $0.84 ; 2.38$ & 0.189 & & & 0.643 \\
\hline$\geq 40$ & 1 & & & 1 & & \\
\hline$<40$ & 1.42 & & & 1.05 & & \\
\hline $\begin{array}{l}\text { Number of hepatic } \\
\text { metastasis }\end{array}$ & & $0.04 ; 0.38$ & 0.042 & & $0.54 ; 0.88$ & 0.027 \\
\hline 1 & 1 & & & 1 & & \\
\hline $2-3$ & 0.94 & & & 1.07 & & \\
\hline$\geq 4$ & 1.2 & & & 1.23 & & \\
\hline $\begin{array}{l}\text { Perioperative } \\
\text { chemotherapy }\end{array}$ & & $0.67 ; 1.48$ & 0.998 & & $0.66 ; 1.74$ & 0.789 \\
\hline Yes & 1 & & & 1 & & \\
\hline No & 1 & & & 1.07 & & \\
\hline Targeted therapy & & $0.76 ; 1.46$ & 0.747 & & $0.64 ; 1.66$ & 0.898 \\
\hline Yes & 1 & & & 1 & & \\
\hline No & 0.95 & & & 1.03 & & \\
\hline Disease-free interval & & $0.86 ; 0.94$ & 0.045 & & $0.83 ; 0.94$ & 0.031 \\
\hline$\geq 18$ months & 1 & & & 1 & & \\
\hline$<18$ months & 1.02 & & & 1.2 & & \\
\hline
\end{tabular}

\section{Author contributions}

R Haifa and L Soumaya conceived of the presented idea. R Haifa developed the theory and performed the computations. R Haifa and $L$ Soumaya verified the analytical methods. M Nesrine encouraged $\mathrm{H}$ Haifa to investigate and supervised the findings of this work. All authors discussed the results and contributed to the final manuscript. R Haifa wrote the manuscript with support from B Hamouda, R Haifa, M Nesrine, and B Hamouda contributed to the final version of the manuscript.

Financial \& competing interests disclosure

The authors have no relevant affiliations or financial involvement with any organization or entity with a financial interest in or financial conflict with the subject matter or materials discussed in the manuscript. This includes employment, consultancies, honoraria, stock ownership or options, expert testimony, grants or patents received or pending, or royalties.

No writing assistance was utilized in the production of this manuscript.

\section{Ethical conduct of research}

The authors state that they have obtained appropriate institutional review board approval or have followed the principles outlined in the Declaration of Helsinki for all human or animal experimental investigations. In addition, for investigations involving human subjects, informed consent has been obtained from the participants involved.

\section{Open access}

This work is licensed under the Attribution-NonCommercial-NoDerivatives 4.0 Unported License. To view a copy of this license, visit http://creativecommons.org/licenses/by-nc-nd/4.0/

\section{References}

Papers of special note have been highlighted as: • of interest; $\bullet \bullet$ of considerable interest

1. Missaoui N, Jaidaine L, Abdelkader et al. Colorectal cancer in central Tunisia: increasing incidence trends over a 15-year period. Asian Pac. J. Cancer Prev. 12(4), 1073-1076 (2011).

- Relates to the incidence of colorectal cancer (CRC) in Tunisia. 
2. August DA, Ottow RT, Sugarbaker Ph et al. Clinical perspectives on human colorectal cancer metastases. Cancer Metastasis Rev. 3 , 303-324 (1984).

3. Galandiuk S, Wieand HS, Moertel CG et al. Patterns of recurrence after curative resection of carcinoma of the colon and rectum. Surg. Gynecol. Obstet. 174, 27-32 (1992).

4. Guerrera F, Falcoz PE, Renaud S et al. Does perioperative chemotherapy improve survival in patients with resectable lung metastases of colorectal cancer? Interact. Cardiovasc. Thorac. Surg. 24(5), 789-791 (2017).

-. Describes the role of perioperative chemotherapy in improving survival in patients with resectable lung metastases from CRC.

5. Allard MA, Adam R, Giuliante F et al. Long-term outcomes of patients with 10 or more colorectal liver metastases. Br. J. Cancer 117 (5), 604-611 (2017).

- Highlights the need to undergo surgery each time it is possible.

6. Weledji EP. Centralization of liver cancer surgery and impact on multidisciplinary teams working on Stage IV colorectal cancer. Oncol. Rev. 11(2), 331 (2017).

- Describes the importance of working in multidisciplinary teams to treat patients with CRC.

7. Schiergens TS, von Einem J, Thomas MN et al. Multidisciplinary treatment of colorectal liver metastases. Minerva Med. 108(6), 527-546 (2017).

-. Describes the importance of a multidisciplinary approach to the treatment of patients with Stage 4 CRC.

8. Bellier J, De Wolf J, Hebbar M et al. Repeated resections of hepatic and pulmonary metastases from colorectal cancer provide long-term survival. World J. Surg. 42(4), 1171-1179 (2017).

-• Explains the role of repeated resections of hepatic and pulmonary metastases from CRC to provide long-term survival.

9. Adam R, Yi B, Innominato PF. Liver Met Survey International Contributing Centers. Resection of colorectal liver metastases after second-line chemotherapy: is it worthwhile? A Liver Met Survey analysis of 6415 patients. Eur. J. Cancer 78, 7-15 (2017).

10. Macherey S, Bruns C, Alakus H et al. Lung metastasectomy in pulmonary metastatic colorectal carcinoma. Zentralbl. Chir. 143(2), 193-204 (2017).

-• Describes the particularities in the treatment of lung metastases in pulmonary metastatic colorectal carcinoma.

11. Gough DB, Donohue JH, Trastek VA et al. Resection for hepatic and pulmonary metastases in patients with colorectal cancer. Br. J. Surg. 81, 94-96 (1994).

12. Stefan Limmer, Elisabeth Oevermann, Claudia Killaitis et al. Sequential surgical resection of hepatic and pulmonary metastases from colorectal cancer. Langenbecks Arch. Surg. 395 (8), 1129-1138 (2001).

13. Hermanek P, Sobin LH (Eds). UICC TNM Classification of Malignant Tumors. (4th Ed.) 2nd Revision. Springer, Berlin, 1992.

14. Bramhall SR, Gur U, Coldham C et al. Liver resection for colorectal metastases. Ann. R Coll. Surg. Engl. 85(5), 334-339 (2003).

15. Scheele J, Stangl R, Altendorf-Hofmann A et al. Resection of colorectal liver metastases World J. Surg. 19, 59-71 (1995).

16. Inoue M, Ohta M, Iuchi K. Thoracic Surgery Study Group of Osaka University Benefits of surgery for patients with pulmonary metastases from colorectal carcinoma. Ann. Thorac. Surg. 78(1), 238-244 (2004).

17. Saito Y, Omiya H, Kohno K et al. Pulmonary metastasectomy for 165 patients with colorectal carcinoma: a prognostic assessment. J. Thorac. Cardiovasc. Surg. 124 (5), 1007-1013 (2002).

18. Pfannschmidt J, Muley T, Hoffmann $\mathrm{H}$ et al. Prognostic factors and survival after complete resection of pulmonary metastases from colorectal carcinoma: experiences in 167 patients. J. Thorac. Cardiovasc. 126, $732-739$ (2003).

19. Pfannschmidt J, Dienemann H, Hoffmann $\mathrm{H}$ et al. Surgical resection of pulmonary metastases from colorectal cancer: a systemic review of published series. Ann. Thorac. Surg. 84(1), 324-338 (2007).

20. Elias D, Liberale G, Vernerey D et al. Hepatic and extrahepatic colorectal metastases: when resectable, their localization does not matter, but their total number has a prognostic effect. Ann. Surg. Oncol. 2, 900-909 (2005).

21. Avital I, De Matteo R. Combined resection of liver and lung metastases for colorectal cancer. Thorac. Surg. Clin. 16(2), 145-155 (2006).

22. Ambiru S, Miyazaki M, Ito $\mathrm{H}$ et al. Resection of hepatic and pulmonary metastases in patients with colorectal carcinoma. Cancer 82(2), 274-278 (1998).

23. Hamy A, Baron O, Bennouna J et al. Resection of hepatic and pulmonary metastases in patients with colorectal cancer. Am. J.Clin. Oncol. 24(6), 607-609 (2001).

24. Regnard JF, Grunenwald D, Spaggiari L et al. Surgical treatment of hepatic and pulmonary metastases from colorectal cancers. Ann. Thorac. Surg. 66(1), 214-218 (1998).

25. Vogt P, Raab R, Ringe B et al. Resection of synchronous liver metastases from colorectal cancer. World J. Surg. 15 (1), $662-675$ (1991).

26. Van Haltreen HK, Van Geel AN, Hart AA et al. Pulmonary resection for metastases for metatstases of colorectal origin. Chest 107(6), 1526-1531 (1995).

27. Headrick JR, Miller DL, Nagorney DM et al. Surgical treatment of hepatic and pulmonary metastases from colon cancer. Ann. Thorac. Surg. 71(3), 97 (2001). 
28. Poston GJ, Adam R, Alberts S et al. A strategy for improving respectability with curative intent in metastatic colorectal cancer. J. Clin. Oncol. 23(28), 7125-7134 (2005).

29. Imai $\mathrm{K}$, Allard MA, Castro Benitez $\mathrm{C}$ et al. Long-term outcomes of radiofrequency ablation combined with hepatectomy compared with hepatectomy alone for colorectal liver metastases Br. J. Surg. 104(5), 570-579 (2017).

30. Kazunari Sasaki, Georgios A. Margonis et al. Combined resection and RFA in colorectal liver metastases: stratification of long-term outcomes J. Surg. Res. 206 (1), 182-189 (2016).

-• Describes the provision of long-term survival by combined resection and radiofrequency ablation.

31. Tzolii G, Adam. R, Viganò. L et al. Surgery of colorectal liver metastases: pushing the limits Liver Cancer 6(1), 80-89 (2016).

32. Van Cutsem E, Cervantes A, Adam R. ESMO consensus guidelines for the management of patients with metastatic colorectal cancer. Ann. Oncol. 27(8), 1386-1422 (2016).

-• Summarizes recent guidelines for the management of patients with metastatic CRC. 
